\title{
The acceleration of DNA may generate cancer
}

\author{
Alireza Sepehri ${ }^{1}$, Somayyeh Shoorvazi ${ }^{2}$ \\ ${ }^{1}$ Faculty of Physics, Shahid Bahonar University, Kerman, Iran \\ ${ }^{2}$ Islamic Azad University, Neyshabur branch, Neyshabur, Iran
}

\section{Email address:}

A.Sepehri14@gmail.com (A. Sepehri), S.Shoorvazi14@gmail.com (S. Shoorvazi)

\section{To cite this article:}

Alireza Sepehri, Somayyeh Shoorvazi. The Acceleration of DNA may Generate Cancer, European Journal of Biophysics. Vol. 1, No. 2 , 2013, pp. 16-21. doi: 10.11648/j.ejb.20130102.11

\begin{abstract}
In this research, the possibility of generating cancer due to acceleration of DNA is investigated. It is observed that the entanglement between p53 sequence and CG pair is decreased with increasing acceleration .Instead, the entanglement between p53 gene and TA pair is increased with increasing acceleration. Thus the acceleration of p53 leads to mutations from $\mathrm{C}$ to $\mathrm{T}$ and causes various types of cancer. Also the entanglement between the existence of AT and nonexistence of AU pairs in non inertial frames is decreased. It's concluded that some extra AU pairs maybe produce in DNA structures and cause to cancer or DNA destroy .Furthermore the probability for a site being occupied is increased due to acceleration and more information is transcribed of DNA to protein. It maybe disrupts the cellular dynamics and can lead to cancer. Finally the production cross section for hole in DNA is calculated. Unfortunately information transformation from inside to outside of holes isn't complete. The proteins outside this hole don't access to inside the DNA hole and information is lost.
\end{abstract}

Keywords: Acceleration, DNA, Cancer

\section{Introduction}

It is well known that practically all genetic information in living systems is contained in the deoxyribonucleic acid (DNA) sequence. The DNA is packaged into chromosome which is localized in the nucleus of the eukaryotic cells. The famous double-helix structure of DNA is made of two strands that carry organic bases that are bound in pairs by hydrogen bonds, keeping the two strands together. There are 4 types of bases, called A, T, G, C, but only AT and GC pairs are part of the structure of DNA. The AT pairs are bound by two hydrogen bonds, while the stronger GC pairs are bound by three hydrogen bonds[1]. The passage of its gene information to protein, called gene expression, performs by the messenger ribonucleic acid (mRNA), which is usually a single polynucleotide chain. RNA molecules carry the nucleotide bases - A, U, C, G-with U very similar in chemical structure to T. A-U pairing is as strong as A-T pairing[2].

In this research, suggesting the mathematical model for DNA in biological field theory, we investigate the effects of acceleration on DNA structures. A uniformly accelerated DNA is unable to access information about the whole of space-time since, from his perspective, a communication horizon appears. This can result in a loss of information and a corresponding degradation of entanglement between base pairs in DNA structures. Information loss disrupts the cellular dynamics and can lead to serious defects, including cancer.

The outline of the paper is as follows. In section II we show that the acceleration of p53 leads to mutations from CG to TA base pairs in DNA. In section III we discuss the production extra base pairs in accelerating DNA. In section IV we study the effect of acceleration on the probability of a site being occupied. In section $\mathrm{V}$ we obtain the production cross section for hole in DNA. The last section is devoted to summary.

\section{The Acceleration of P53 DNA}

The p53 DNA is said to be the "guardian of the genome" since it encodes the TP53 protein that suppresses the tumor development by activating the DNA repair mechanisms or the cell apoptosis process if the damage of DNA is irreparable. More than $50 \%$ of human cancers are related to the mutations of the p53 gene which jeopardize the efficient functioning of TP53[3]. Most of the cancerous mutations are point mutations - a base pair substituted by another with distributions along the DNA sequence that are highly non-uniform[4].The positions where the mutations occur 
most frequently are call the "hotspots" of mutations[5]. The 14585th base pair of the p53 sequence is a particularly active hotspot with 133 entries in the IARC database [4]. It exhibits mutations from $\mathrm{C}$ to $\mathrm{T}$ and causing various types of cancer. However, the mutations $\mathrm{C} \rightarrow \mathrm{G}$ and $\mathrm{C} \rightarrow \mathrm{A}$ at the same position are not cancerous [5].

Now we discuss the effect of acceleration on point mutations. Previously the entanglement between two modes of the free scalar and Dirac fields as seen by two relatively accelerated observers has been investigated [6-8]. It is found that a state which is maximally entangled in an inertial frame becomes less entangled if the observers are relatively accelerated. Using these calculations we can discuss the effect of acceleration on the entanglement between base pairs in DNA structures.

Consider DNA to be uniformly accelerated in the $(t$, z)plane $(\mathrm{c}=1)$ (figure1.). Rindler coordinates $(\tau, \xi)$ are appropriate for describing the viewpoint of an observer moving with uniform acceleration. Two different sets of Rindler coordinates, which differ from each other by an overall change in sign, are necessary for covering Minkowski space. These sets of coordinates define two Rindler regions that are causally disconnected from each other[6,7].

$$
\begin{array}{lll}
a t=e^{a \xi} \sinh (a \tau) & , a z=e^{a \xi} \sinh (a \tau), & \text { in region I } \\
a t=-e^{a \xi} \sinh (a \tau) & , a z=-e^{a \xi} \sinh (a \tau), & \text { in region II }
\end{array}
$$

where a denotes DNA's acceleration. The above set of coordinates both give rise to the same Rindler metric:

$$
d s^{2}=d t^{2}-d z^{2}-d^{2} x_{\perp}=e^{2 a \xi}\left(d^{2} \tau-d^{2} \sigma\right)-d^{2} x_{\perp}
$$

where $x_{\perp}=(x, y)$ are the same in both Minkowski and Rindler space-times. A DNA undergoing eternal acceleration remains constrained to either Rindler region I or II and has no access to the opposite region, since these two regions are causally disconnected.

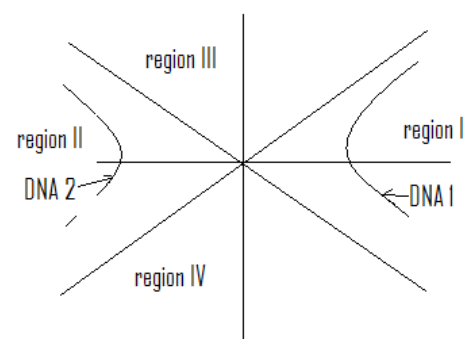

Figure 1. Spacetime diagram, showing the trajectories of an inertial and an accelerated observer [7].

The uniform acceleration consists first in duplicating the degrees of freedom of the system. To this end a copy of the original Hilbert space may be constructed with a set of

$$
C_{C G}\left|D N A, C G_{0}\right\rangle_{I \otimes I I}=\left(\cosh r \alpha_{C G}^{I}-\sinh r \alpha_{T A}^{I I}\right)\left|D N A, C G_{0}\right\rangle_{I \otimes I I}=0
$$

where CCG is annihilation operator in Minkowski vacuum. We can rewrite equation (4)as following:

$$
C_{C G}\left|D N A, C G_{0}\right\rangle_{I \otimes I I}=\left(\cosh r \frac{\partial}{\partial \alpha_{C G}^{\dagger I}}-\sinh r \alpha_{T A}^{I I}\right)\left|D N A, C G_{0}\right\rangle_{I \otimes I I}=0
$$

The solution of this equation is given by

$$
\left|D N A, C G_{0}\right\rangle_{I \otimes I I}=e^{\tanh r \alpha_{C G}^{I \dagger}, \alpha_{T A}^{I \dagger \dagger}}|0\rangle_{S} \approx \frac{1}{\cosh r} \sum_{n=0}^{\infty} \tanh ^{n} r\left|D N A, C G_{n}\right\rangle^{I}\left|D N A, T A_{n}\right\rangle^{I I}
$$

where $\left|D N A, C G_{0}\right\rangle_{I \otimes I I}$ is vacuum state for CG pair in
Minkowski space-time and $\left|D N A, C G_{n}\right\rangle^{I},\left|D N A, T A_{n}\right\rangle^{I I}$ are orthonormal bases (normal mode solutions) for $n \mathrm{CG}$ 
and TA pairs in HI and HII respectively. We assume that space-time. In this case we get: before acceleration there is one CG pair in Minkowski

$$
\begin{aligned}
& \left|D N A, C G_{1}\right\rangle_{I \otimes I I}=C_{C G}^{\dagger}\left|D N A, C G_{0}\right\rangle_{I \otimes I I} \\
& =\left(\cosh r \alpha_{C G}^{I}-\sinh r \alpha_{T A}^{I I}\right) \frac{1}{\cosh r} \sum_{n=0}^{\infty} \tanh ^{n} r\left|D N A, C G_{n}\right\rangle^{I}\left|D N A, T A_{n}\right\rangle^{I I} \\
& =\frac{1}{\cosh ^{2} r} \sum_{n=0}^{\infty} \tanh ^{n} r \sqrt{n+1}\left|D N A, C G_{n+1}\right\rangle^{I}\left|D N A, T A_{n}\right\rangle^{I I}
\end{aligned}
$$

We can do similar calculation forp53 sequence :

$$
\begin{gathered}
\left.\left.\left.\mid D N A, p 53 \text { sequence }_{0}\right\rangle_{I \otimes I I} \approx \frac{1}{\cosh r} \sum_{n=0}^{\infty} \tanh ^{n} r \mid D N A, p 53 \text { sequence }_{n}\right\rangle^{I} \mid D N A, p 53 \text { sequence }_{n}\right\rangle^{I I} \\
\left.\mid D N A, p 53 \text { sequence }_{1}\right\rangle_{I \otimes I I}= \\
\left.\left.=\frac{1}{\cosh ^{2} r} \sum_{n=0}^{\infty} \tanh ^{n} r \sqrt{n+1} \mid D N A, p 53 \text { sequence }_{n+1}\right\rangle^{I} \mid D N A, p 53 \text { sequence }_{n}\right\rangle^{I I}
\end{gathered}
$$

Consider that CG pair and p53 sequence in Minkowski perspective, i.e., the quantum field is in a state. space-time are maximally entangled from an inertial

$$
\left.\left.\frac{1}{\sqrt{2}}\left(\mid D N A \text {, sequence } p 53_{0}\right\rangle^{M}\left|D N A, C G_{0}\right\rangle^{M}+\mid D N A \text {, sequence } p 53_{1}\right\rangle^{M}\left|D N A, C G_{1}\right\rangle^{M}\right)
$$

The states

$\mid D N A$, sequence $\left.p 53_{0}\right\rangle^{M}, \mid D N A$, sequence $\left.p 53_{1}\right\rangle^{M} \quad$ are the vacuum and single DNA sequence states and the states $\left|D N A, C G_{0}\right\rangle^{M},\left|D N A, C G_{1}\right\rangle^{M}$ are the vacuum and single CG states in Minkowski space .

If DNA undergoes uniform acceleration a,the states

$$
\begin{aligned}
& \rho_{p 53 \text { sequence }, C G}=\frac{1}{2 \cosh ^{4} r} \sum_{n}(\tanh r)^{4 n} \rho_{n} \\
& \left.\rho_{n}=\mid p 53 \text { sequence }_{n}, C G_{n}\right\rangle\left\langle p 53 \text { sequence }_{n}, C G_{n}\right| \\
& \left.+\frac{n+1}{\cosh ^{2} r} \mid p 53 \text { sequence }_{n}, C G_{n}\right\rangle\left\langle p 53 \text { sequence }_{n+1}, C G_{n+1}\right| \\
& \left.+\frac{n+1}{\cosh ^{2} r} \mid p 53 \text { sequence }_{n+1}, C G_{n+1}\right\rangle\left\langle p 53 \text { sequence }_{n}, C G_{n}\right| \\
& \left.+\left(\frac{n+1}{\cosh ^{2} r}\right)^{2} \mid p 53 \text { sequence }_{n+1}, C G_{n+1}\right\rangle\left\langle p 53 \text { sequence }_{n+1}, C G_{n+1}\right|
\end{aligned}
$$

To obtain the amount ofentanglement between p53 sequence and $\mathrm{CG}$ pair, we can use of logarithmic negativity[6-8].Previously the amount of entanglement for two relatively accelerated scalar fields is calculated[6].Doing similar calculations, we get: 


$$
\begin{gathered}
N\left(\rho_{p 53 \text { sequence, } C G}\right)=\log _{2}\left(\sum _ { n = 0 } ^ { \infty } \frac { \operatorname { t a n h } ^ { 4 n } r } { 2 \operatorname { c o s h } ^ { 4 } r } \left(\left(\frac{n+1}{\cosh ^{2} r}\right)^{2}+\sqrt{\left.\left.\left(\frac{n+1}{\cosh ^{2} r}\right)^{2}+4 \frac{n+1}{\cosh ^{2} r}\right)\right)}\right.\right. \\
= \begin{cases}1 & \text { for } a \rightarrow 0 \\
0 & \text { for } a \rightarrow \infty\end{cases}
\end{gathered}
$$

We observe that for vanishing acceleration, negativity is unity and for inifinity acceleration the negativity is exact zero. This means that entanglement between $\mathrm{p} 53$ sequence and CG is decreased with increasing acceleration .Instead with similar calculations we can show that entanglement between p53 sequence and TA pair is increased with increasing acceleration. It's conclude that the acceleration ofp53 leads to mutations from $\mathrm{C}$ to $\mathrm{T}$.

\section{The Mutations from $T$ to $U$ in Accelerating DNA}

Now we discuss the effect of acceleration on the mutations from $\mathrm{T}$ to $\mathrm{U}$ in DNA. In Minkowski space, DNA and mRNA carry AT pairs and AU respectively. Thus we can consider the entanglement between existence of AT and non-existence of AU pairs in DNA in inertial frame,

$$
\frac{1}{\sqrt{2}}\left(\left|A T_{0}\right\rangle^{M}\left|A U_{1}\right\rangle^{M}+\left|A T_{1}\right\rangle^{M}\left|A U_{0}\right\rangle^{M}\right)
$$

$\left|A T_{0}\right\rangle^{M},\left|A T_{1}\right\rangle^{M}$ are the vacuum and single particle excitation states of the AT base and $\left|A U_{0}\right\rangle^{M},\left|A U_{1}\right\rangle^{M}$ are the vacuum and single particle excitation states of the $\mathrm{AU}$ base in Minkowski space.

The Minkowski vacuum state of AT and AU pairs, can be expressed in terms of a product of two-mode squeezed states of the Rindler vacuum [6]

$$
N\left(\rho_{A T, A U}\right)=\log _{2}\left(\frac{1}{2 \cosh ^{4} r} \sum_{n}(\tanh r)^{4 n}(n+1)\right)= \begin{cases}1 & \text { for } a \rightarrow 0 \\ 0 & \text { for } a \rightarrow \infty\end{cases}
$$

In an inertial frame, $\mathrm{N}=1$, and the state of the system defined by Eq. (19) is maximally entangled. In the limit of infinite acceleration, the logarithmic negativity is zero implying that the entanglement in the infinite acceleration limit is vanishes. This means that the entanglement between existence of AT and non-existence of AU pairs in DNA in non inertial frames is decreased. It's concluded that some extra AU pairs maybe form in DNA structures .

\section{The Effect of Acceleration on the Probability of a Binding Site Being Occupied}

The primary step in the production of a protein is the transcription of a gene from the DNA template to a

$$
\begin{aligned}
& \left|A T_{0}\right\rangle_{I \otimes I I} \approx \frac{1}{\cosh r} \sum_{n=0}^{\infty} \tanh ^{n} r\left|A T_{n}\right\rangle^{I}\left|A T_{n}\right\rangle^{I I} \\
& \left|A U_{0}\right\rangle_{I \otimes I I} \approx \frac{1}{\cosh r} \sum_{n=0}^{\infty} \tanh ^{n} r\left|A U_{n}\right\rangle^{I}\left|A U_{n}\right\rangle^{I I}
\end{aligned}
$$

$$
\begin{gathered}
\left|A T_{1}\right\rangle_{I \otimes I I}=\frac{1}{\cosh ^{2} r} \sum_{n=0}^{\infty} \tanh ^{n} r \sqrt{n+1}\left|A T_{n+1}\right\rangle^{I}\left|A T_{n}\right\rangle^{I I} \\
\left|A U_{1}\right\rangle_{I \otimes I I}=\frac{1}{\cosh ^{2} r} \sum_{n=0}^{\infty} \tanh ^{n} r \sqrt{n+1}\left|A U_{n+1}\right\rangle^{I}\left|A U_{n}\right\rangle^{I I}
\end{gathered}
$$

We can rewrite Eq. (13) in terms of Rindler modes for AT and AU pairs. Since these pairs are causally disconnected from region II, we will trace over the states in this region and obtain

$$
\rho_{A T, A U}=\frac{1}{2 \cosh ^{4} r} \sum_{n}(\tanh r)^{4 n}(n+1) \rho_{n}
$$

$$
\begin{aligned}
& \rho_{n}=\left|A T_{n+1}, A U_{n}\right\rangle\left\langle A T_{n+1}, A U_{n}|+| A T_{n+1}, A U_{n}\right\rangle\left\langle A T_{n}, A U_{n+1}\right| \\
& +\left|A T_{n+1}, A U_{n}\right\rangle\left\langle A T_{n}, A U_{n+1}|+| A T_{n}, A U_{n+1}\right\rangle\left\langle A T_{n}, A U_{n+1}\right|
\end{aligned}
$$

To obtain the amount of entanglement between existence and non-existence of AT and AU pairs, we can use of logarithmic negativity[6].With similar calculations to ref[6] we get
m(essenger)-RNA molecule by activating RNA-polymerase by transcription factors. Transcription factors are proteins which bind to specific sites on DNA. These binding sites are located in the so-called regulatory region of a gene, which for yeast typically extends over several hundred nucleotides before the starting point of transcription .A fully assembled set of transcription factors will attract and activate the machinery (called RNA-polymerase) which transcribes the DNA template to an mRNA molecule. When one site is occupied by transcription factors, the regulated gene will get transcribed into mRNA[11].

Assuming that a transcription factor may either be bound at a binding site, or be suspended in solution or bound nonspecifically elsewhere on DNA, the probability of a given site being occupied is given by elementary statistical mechanics $[11,12]$ as 


$$
n_{\text {occupied }}=\frac{n_{1 \text { protein }} e^{-\beta E}}{n_{1 \text { protein }} e^{-\beta E}+e^{-\beta F}}
$$

this probability depends on the transcription factor concentrationn1protein,binding energy $\mathrm{E}$ and a free energy F of the transcription factor in solution or bound elsewhere.

According to Unruh idea, when a DNA undergoes a uniform proper acceleration a, perceives a thermal distribution of proteins[7]. Thus the DNA appears to be in a heat bath at the Unruh temperature. In this condition,the Minkowski vacuum state of proteins, can be expressed in terms of a product of two-mode squeezed states of the Rindler vacuum[6]

$$
\left.\left.\mid \text { protein }_{I \otimes I I} \approx \frac{1}{\cosh r} \sum_{n=0}^{\infty} \tanh ^{n} r \mid \text { protein }_{n}\right\rangle^{I} \mid \text { protein }_{n}\right\rangle^{I I}
$$

We can calculate the thermal distribution ofproteins as following:

$$
\begin{aligned}
& n_{2 \text { protein }}={ }_{I \otimes I I}\left\langle\text { protein }\left|\alpha_{I}^{\dagger \text { protein }} \alpha_{I}^{\text {protein }}\right| \text { protein }_{I \otimes I I}\right. \\
& \left.={ }_{I I}\left\langle\text { protein }\left._{n}\right|_{I}\left\langle\text { protein }_{n}\left|\frac{1}{\cosh ^{2} r} \alpha_{I}^{\dagger \text { protein }} \alpha_{I}^{\text {protein }} \sum_{n=0}^{\infty} \tanh ^{2 n}(r)\right| \text { protein }_{n}\right\rangle_{I}\right| \text { protein }_{n}\right\rangle_{I I} \\
& \left.\left.={ }_{I I}\left\langle\text { protein }\left._{n}\right|_{I}\left\langle\text { protein }_{n-1}\right| \frac{1}{\cosh ^{2} r} \alpha_{I}^{\dagger} \text { protein }^{\text {protein }} \sum_{I}^{\infty} \tanh ^{2 n}(r) n\right| \text { protein }_{n-1}\right\rangle_{I} \mid \text { protein }_{n}\right\rangle_{I I} \\
& =\frac{1}{\cosh ^{2} r_{E, F, y}} \sum_{n=0}^{\infty} \tanh ^{2 n}(r)(n)=\sinh ^{2}(r)=\frac{e^{-\frac{2 \pi \omega}{a}}}{1-e^{-\frac{2 \pi \omega}{a}}}
\end{aligned}
$$

Using the thermal distribution for proteins, we can correct the probability in equation(20):

$$
n_{\text {occupied }}=\frac{\left(n_{1 \text { protein }}+n_{2 \text { protein }}\right) e^{-\beta E}}{\left(n_{1 \text { protein }}+n_{2 \text { protein }}\right) e^{-\beta E}+e^{-\beta F}}
$$

It is found that the probability for a site being occupied is increased due to acceleration and more information is transcribed of DNA to protein. $t$ maybe disrupt the cellular dynamics.

\section{Theproduction Cross Section of Hole in Cell}

If DNA undergoes uniform high acceleration a, maybe one hole forms. Because the energy $\mathrm{E}$ that is performed on the DNA molecule to keep it in uniform acceleration is the product of $\mathrm{F}$ times a characteristic distance through which the force acts, which we take to be the Compton wavelength of the DNA $\delta z=\frac{\hbar}{m c}$. This yields [7]

$$
E=F \delta z=M_{D N A} a \frac{\hbar}{M_{D N A} c}=\frac{\hbar a}{c} \approx k_{B} T
$$

The Unruh temperature of this hole similar to Hawking temperature of black hole can be calculated as following:

$$
T_{U}=\frac{\hbar a}{c k_{B}}
$$

Following the calculations in refs $[13,14]$ the production cross section for this hole can be obtained as:

$$
\begin{aligned}
& \sigma^{\text {Acceleration } \rightarrow D N A-H o l e}=\sum_{a b} \int_{\tau}^{1} d x_{C G} \int_{\frac{\tau}{x_{a}}}^{1} d x_{A T} f_{C G}\left(x_{C G}\right) \\
& \times f_{A T}\left(x_{A T}\right) \frac{1}{M_{D N A}^{2}}\left[\frac{x_{C G} x_{A T} \hbar a}{M_{D N A} c}\left(4 \Gamma\left(\frac{3}{2}\right)\right)\right]^{2} \delta\left(x_{C G} x_{A T}-\frac{M_{D N A-H o l e}^{2}}{\hbar a}\right)
\end{aligned}
$$

Where $\mathrm{xCG}, \mathrm{xAT}$ is the longitudinal momentum fraction of the base-pairs inside the protein , $f_{C G}\left(x_{C G}\right)$ and $f_{A T}\left(x_{A T}\right)$ are the distribution of base pairs in DNA, $M_{D N A-H o l e}, M_{D N A}$ are the hole mass and DNA mass and $\tau=\frac{M_{D N A-H o l e}^{2}}{\hbar a} . \sum_{a b}$ represents the sum over all base-pair contributions.

The proteins carry information that is contained in DNA. Unfortunately information transformation from inside to outside of holes isn't complete. The proteins outside this 
hole don't access to inside the DNA hole and information is lost.

\section{Summary}

In this research, the effect of acceleration on entangled base pairs in DNA structures is studied. The entanglement between p53 sequence and CG pair is decreased with increasing acceleration and the entanglement between p53 gene and TA pair is increased with increasing acceleration. Thus the acceleration of $\mathrm{p} 53$ exhibits mutations from $\mathrm{C}$ to $\mathrm{T}$. Also It's concluded that some extra AU pairs maybe produce in DNA structures due to decrease in entanglement between the existence of AT and non-existence of AU pairs in non inertial frames. Furthermore the probability for a site being occupied is affected by acceleration. It maybe disrupts the cellular dynamics. Finally the production cross section for DNA holes is calculated.

\section{References}

[1] Pui-Man Lam, Yi Zhen, Physical Review E83, 041912 (2011). Michel Peyrard, Santiago Cuesta-Lopez, Dimitar Angelov, Journal of Physics: Condensed Matter 3, 21 (2008) 034103-1-13

[2] Pui-Man Lam, Richard M. Neumann, Physical Review E84, 032901 (2011).Branko Dragovich, Alexandra Dragovich, pAdic Numbers, Ultrametric Analysis and Applications 1 (2009) 34-41
[3] C. J. Sherr, Cell 234, 116 (2004).

[4] A. Petitjean et al., Hum. Mutat. 28, 622 (2007),

[5] Chi-Tin Shih, Stephan Roche, Rudolf A. Römer, Phys. Rev. Lett. 100, 018105 (2008)

[6] I. Fuentes-Schuller and R. B. Mann, Phys. Rev. Lett. 95, 120404 (2005).

[7] P. M. Alsing, I. Fuentes-Schuller, R. B. Mann, and T. E. Tessier, Phys. Rev. A 74, 032326 (2006).

[8] Qiyuan Pan, Jiliang Jing, Phys.Rev.A77:024302,2008

[9] Alireza Sepehri, Somayyeh Shoorvazi. Information Loss in Transcribing Genetic Sequence from DNA into Protein. American Journal of Bioscience and Bioengineering. Vol. 1, No. 2,2013, pp. 43-47. doi: 10.11648/j.bio.20130102.13

[10] Alireza Sepehri, Somayyeh Shoorvazi. The Possibility of Information Loss in Transcribing Genetic Sequence of DNA into Proteins due to Acceleration of Universe. American Journal of Bioscience and Bioengineering. Vol. 1, No. 2, 2013, pp. 38-42.doi: 10.11648/j.bio.20130102.12.

[11] Jose M. G. Vilar and Leonor Saiz,Phys. Rev. Lett. 96, 238103 (2006)

[12] Johannes Berg, revised version to appear in Europhys. Lett.( arXiv: 0712. 3791).Gasper Tkacik and Aleksandra M. Walczak,arXiv: 1101.4240

[13] S. Dimopoulos and G. Landsberg, Phys. Rev. Lett. 87, 161602

[14] S. B. Giddings and S. Thomas, Phys. Rev. D 65, 056010 (2002) 